\title{
Protée
}

\section{La musique de la relation}

\section{Serge Cardinal}

Volume 27, numéro 1, 1999

La Mort de Molière et des autres

URI : https://id.erudit.org/iderudit/030538ar

DOI : https://doi.org/10.7202/030538ar

Aller au sommaire du numéro

Éditeur(s)

Département des arts et lettres - Université du Québec à Chicoutimi

\section{ISSN}

0300-3523 (imprimé)

1708-2307 (numérique)

Découvrir la revue

\section{Citer cet article}

Cardinal, S. (1999). La musique de la relation. Protée, 27(1), 39-42.

https://doi.org/10.7202/030538ar
Résumé de l'article

La Mort de Molière rejoue l'idéal d'une musicalité du cinéma, mais c'est pour mieux le faire filer, le déplacer, le soustraire à un désir de systématicité solitaire et le livrer aux turbulences d'une action, d'une intervention, d'une capture du monde. d'utilisation que vous pouvez consulter en ligne.

https://apropos.erudit.org/fr/usagers/politique-dutilisation/ 


\section{LA MUSIQUE DE LA RELATION}

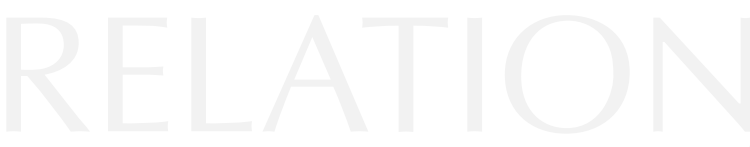

SERGE CARDINAL

La Mort de Molière est tissée de répétitions. Des éléments en série se croisent pour former une trame vidéographique: des bruits, des cris, des onomatopées, des mots, des phrases, des gestes, des costumes, des personnages, des objets, des scènes, des images, des mouvements de caméra, des séquences se répètent, se répondent, se reprennent pour former une "musique», une chaîne rythmique, une multiplicité, une suite de relations rameuses et ramifiantes.

Il ne s'agit donc pas uniquement de répétitions homogènes, statiques et statistiques, de répétitions indifférentes réduites à une distribution illimitée d'éléments semblables extérieurs les uns aux autres. Les éléments indifférents de la répétition matérielle sont au contraire impliqués par une relation. Cette relation crée à la fois quelque chose de nouveau et des degrés variables de cette nouveauté: chaque série d'éléments se laisse entraîner dans des arrangements, des permutations, des combinaisons, des renversements, des transpositions, des variations.

Le trépas de Molière est un passage de la répétition à la relation, de la multiplicité numérique à la multiplicité qualitative, de la mesure au rythme, du collage au montage, de l'espace au temps. En tous ces sens, La Mort de Molière rejoue l'idéal d'une musicalité du cinéma, mais c'est pour mieux le faire filer, le déplacer, le soustraire à un désir de systématicité solitaire et le livrer aux turbulences d'une action, d'une intervention, d'une capture du monde.

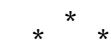

Des pas et des éclats de verre répétés; des énoncés repris et retournés: «Ce n'est pas un poème sur Molière», "Molière n'est pas un sujet de poésie», "C'est un poème sur Molière»; des variations à partir d'un nombre réduit de cellules musicales; des personnages dans un ordre strict passés et repassés en revue; deux religieuses debout, l'une qui s'assoit, puis l'autre, l'une qui croise les bras, puis l'autre aussi; «Euh! Euh! Euh! Brute! Euh! Brute!». La Mort de Molière apparaît d'abord comme un système de renvois d'un signe à l'autre, de reprises d'un signe par l'autre, à l'infini, comme un ensemble de rapports formels redondants où le 
spectateur ne cesse de retrouver ce qui avait momentanément disparu; comme si, dans une gigantesque et puissante circularité, tout signe était condamné au retour, tel un mort revenant hanter le nouveau souffle, le dernier circuit de la perception et de l'interprétation, le spectateur ne finissant jamais de payer sa dette à la gloire d'un système signifiant.

Toujours un signe derrière un signe: circularité et recyclage. Tout est rejoué, tout est répétition dans ce monde endeuillé. On pourrait même croire que c'est elle, la répétition, qui tue. Pourtant:

Ils le conjurèrent, les larmes aux yeux, de ne point jouer ce jourlà, et de prendre du repos pour se remettre. "Comment voulezvous que je fasse; leur ditil; il y a cinquante pauvres ouvriers qui n'ont que leur journée pour vivre; que feront-ils, si l'on ne joue pas? Je me reprocherais d'avoir négligé de leur donner $d u$ pain un seul jour, le pouvant faire absolument».

La répétition, l'alignement des corps, éternellement et infiniment repris, ne donnerait qu'un collage de dépouilles, mais s'il est question de jeu et de musique, c'est qu'il est question de montage, de relations, de transformations incorporelles attribuées à des corps. Ces transformations incorporelles ne font pas d'un corps mourant un malade imaginaire; elles sont des actions réelles d'une pensée qui affronte le temps turbulent de la mort, le temps hors de ses gonds. Le collage des corps - des images, des sons, des mouvements, des mots, etc. -, l'action sensible d'un corps sur l'autre appelle une contrepartie: des relations spirituelles. Et ces relations, effectuant des opérations sur ces corps, font d'une ressemblance et d'une contiguité perceptives un principe d'association qui excède le donné sensible, qui excède d'un élan vital la chronologie des corps et saute dans le rythme démentiel de la pensée: «à quatre heures précises", des possibilités de vie défilent devant vos yeux, le possible est rejoué, c'est-à-dire créé, la mort vous donne la dernière chance de penser l'impensable: ce qu'il y a de création incorporelle qui échappe à l'épuisement des combinaisons corporelles. Donnant le matériau, le collage des images est cause de la relation. Mais rien n'est dit encore de la condition de cette relation: ce qui est attribué aux corps est en réalité un sentiment de nécessité dans l'esprit, une croyance, une foi; la répétition a besoin d'une association dans l'esprit, d'un montage dans l'imagination capable de faire événement d'une rencontre entre des corps, nous donnant ainsi des raisons de croire au monde. Cette croyance est paradoxale; elle n'est pas égale à la reconnaissance de formes achevées par un sujet constitué. Si Molière exige de répéter, de rejouer, c'est justement parce qu'il n'y a rien de définitif dans cette croyance, elle est toujours au seuil de la mort, sur le point de perdre le monde dans le cliché ou le stéréotype, dans la répétition nue. Pour Molière, la répétition reste toujours la dernière chance d'une relation, d'un événement qui est à la fois affection d'un spectateur et création d'un monde, un instant, à la limite du vivable, où la chaîne associative cesse de rassembler des contenus conventionnels pour un individu déterminé, mais devient principe impersonnel de création du spectateur, de la scène et du spectacle. L'esprit affronte La Mort de Molière: la pensée y naît comme le monde de la relation créée entre les corps répétés, dans cette dimension supplémentaire à la limite des corps, dans cette implication réciproque qui est tout sauf une homogénéisation. Si bien qu'on ne peut pas s'en tenir à la perception: d'une collection dans l'espace, il faut passer à une association dans le temps; de la mesure, il faut tirer du rythme, des différences de potentiel qui sont autant de germes de vie pour la pensée et pour un monde.

La relation est donc une fuite, fuite en tant que dérive, échappée, conquête. Sortir ainsi de la mesure, c'est se dégager d'un certain type d'individuation de structure ou de concept, c'est s'échapper du tombeau et s'engager sur des associations, des lignes associatives qui ne rebroussent plus vers une totalité d'origine ou ne poussent plus vers une totalité de destination, fussent-elles, justement, toujours différées ou manquées. De la répétition naît une puissance de relation non réductible au nombre possible des combinaisons à l'intérieur d'un système. Au contraire, la ligne de relation capte des domaines différents et y 
effectue des changements incorporels qui sont autant d'événements singuliers. Cette capture de l'hétérogène est une sortie de la musique par la musique, du cinéma par le cinéma: La Mort de Molière met en jeu des variables d'expression qui sont autant de raisons internes de ne pas se fermer sur soi; elle développe une puissance de relation qui a besoin d'ouvrir sur le dehors pour tenter un acte, pour capturer le monde. Non plus représenter, mais repousser, anticiper, ralentir, précipiter, détacher ou réunir les corps les plus hétérogènes du monde, une manière de s'insérer dans le monde qui ne serait ni représentative, ni informative, ni communicationnelle, mais effective. Des changements incorporels: des opérations, des effets. C'est pourquoi, si le mélange des corps dans La Mort de Molière donne l'impression d'une autosuffisance, d'une fermeture dans la redondance, il n'en contient pas moins, précisément par cette constance et cette rigueur de la répétition, une capacité d'ébranler l'esprit, de le forcer, de le mobiliser, d'y injecter le sentiment de la relation, une affection, une musicalité qui n'a plus simplement à voir avec le fait musical au sens strict, mais avec une tendance de l'esprit à associer, association d'une puissance en vue d'une joie. En ce sens, invoquer une musicalité du cinéma n'équivaut pas à définir en quoi le montage visuel imite la musique. Le matériau visuel n'est plus une possibilité de représentation de la musique, mais une possibilité de mise en relation et de capture d'une variable. Il n'y a pas imitation ou emprunt à la musique par le cinéma de procédures qui deviendraient communes, mais des moyens différents de mettre en relation pour capter la même chose: le temps, le temps perdu, le temps retrouvé.

On l'aura compris, la musicalité ici est "volonté" de relation. Elle n'est pas une mécanique céleste ni une mathématique du temps. Elle a quitté définitivement le monde des correspondances pythagoriciennes et spirituelles. Elle n'est pas non plus ce système combinatoire, ce régime de signes où le son en soi et pour soi se déploie dans un jeu de renvois intra et intermusicaux. Non pas que tout cela soit trop abstrait, au contraire; abstraite, la structure ne l'est jamais assez. Car ce qu'exprime la musicalité de Molière - et au demeurant ce qu'exprime la musique à la énième puissance-, ce n'est pas l'idéalité d'une structure, c'est une abstraction plus grande encore, mais non moins réelle, c'est un principe d'association, c'est une volonté impersonnelle de relation. Opérant par matières et fonctions, cette machine relationnelle abstraite ne fait pas de différence entre l'expression et le contenu. La croyance en ce monde-ci est à ce prix: La Mort de Molière traverse autant les os que la langue, la chair que des concepts; passer, telle est son affaire, passer du physique au politique à l'historique au rhétorique... La croyance est au bout de ces séries divergentes et de ces ensembles incompossibles.

Non, la musique n'est pas un «jardin de verre géométrique». La musique arrive alors qu'on se dégage de la mesure et de la partition pour atteindre le rythme; à tout coup «le résultat dépend d'autres circonstances que le cours des étoiles». Et le rythme ne s'effectue pas au même niveau que le rythmé; la relation est extérieure aux termes répétés. Le rythme, c'est un «mouvement au travail»: «Father, I am dying. Father, I am dying. Father, I am dying...», c'est un météore qui crée une transversale sur la carte du ciel. La musique n'est pas collective mais distributive, il n'y a pas de combinaison capable de comprendre tous les éléments; il n'y a que des conjonctions conquises: des alternances et des entrelacements, des attractions et des distractions. La musique est puissance de relation, «une volonté de répandre la semence là où tend la violence du désir». Ce n'est jamais associer toutes les choses à la fois, ni toutes les choses d'une seule fois, mais une à une toutes les fois: "le sang jaillit dans la direction de qui a frappé et l'ennemi, s'il s'offre, est couvert de sang». La musique de Molière est capture de l'hétérogène: Lautréamont, Corneille, Shakespeare, Kafka, Lucrèce, l'argent, l'amérindien, le théâtre, la médecine, les animaux, les insectes, les démons... Et ce n'est jamais qu'un simple mélange des corps.

Il existe une musicalité du cinéma dans la mesure où le spectateur est danseur, dans la mesure où il contre-effectue ce que le mélange effectuait déjà, mais 
dans une dimension supplémentaire, dans une transversale, dans une ligne abstraite. La Mort de Molière invente le spectateur dont elle a besoin, un spectateur impossible, capable des plus grandes torsions, des plus grands sauts, un spectateur d'une légèreté et d'une souplesse telles qu'il ne met jamais le pied sur une articulation mais les emporte toutes dans le mouvement. Son spectateur danse comme Glenn Gould chante, d'un chant qui de vouloir tout parcourir vise l'idée en grognant, en ne soutirant qu'une ligne de traverse qui fend la partition: «one man in his time plays many parts». Son spectateur est «un épouseur à toutes mains» qui danse entre les mesures: d'un «coquillage incrusté dans le crâne» aux "obscénités qui aiguillonnent la chair solitaire», du "débarquement désastreux» jusqu' aux «silhouettes dans les décombres", des "forêts brûlées en Eastman Color» à «Fritz Lang qui étrangle Boris Karloff»... Et comme la musique n'est pas dans la mesure mais dans le rythme, danser ne sera jamais aussi simple que de tenir la cadence: ce qui lie tous ces disparates, ce n'est pas, ce ne peut pas être une identité de structure, mais un vecteur de sortie.

Plus Molière se retire dans la mort, plus son tombeau fuit vers le monde: d'un rapprochement répété entre les visages, de l'accord symétrique d'un geste avec un autre, de la scansion d'une exclamation à la répétition d'un cri, du redoublement des carreaux par les fenêtres, de la femme par l'amante et d'une religieuse par toutes les autres, de "Molière se meurt" à «Molière se meurt», une puissance de relation, une musicalité, se constitue pour aussitôt s'ouvrir sur un dehors qui n'est pas un espace de délire, mais un temps de la pensée où l'éclat des «panneaux de signalisation» s'associe à «l'éclatement des boîtes de bière», où la "traversée des banlieues" par un «troupeau de comédiens" s'associe à «la lente agonie des mouches"... La Mort de Molière s'arrache à la circularité du signe ad nauseam; ici, ce n'est pas en vain que l'on meurt. Entre deux cris - Molière est mort! Vive Molière!-, voilà qu'une mort crée du nouveau, en provoquant la répétition, en précipitant la chronique d'une vie, en l'arrachant à l'égrènement nécrologique des états civils. La répétition est l'occasion du possible; la relation n'est jamais simplement l'épuisement des combinaisons. Il faut jouer encore et toujours! Non pas jusqu'à la fin, mais en extrayant de la fin une fuite, de la création, des mots de passe, des passages par où l'on pourrait croire au monde. Jamais l'immortalité, toujours la métamorphose. Échapper au ressassement éternel, mourir dans un dernier acte dont les conséquences nous échappent, dont l'affirmation reste libre de notre arrêt de mort. Mourir ad libitum.

Note: Toutes les citations proviennent des textes de la vidéo, écrits et choisis par Heiner Müller.

\section{RÉFÉRENCES BIBLIOGRAPHIQUES}

BRÉHIER, É. [1997]: La Théorie des incorporels dans l'ancien stoïcisme, Paris, Librairie philosophique J. Vrin.

Deleuze, G. [1969] : Logique du sens, Paris, Minuit; [1980]: Mille plateaux, Paris, Minuit; [1985]: L'Image-temps, Paris, Minuit. EISENSTEIN, S. [1976]: La Non-indifférente nature, tome 1, Paris, 10/18. Hume, D. [1978]: A Treatise of Human Nature, Oxford, Clarendon Press. 